\title{
A Bailout of Iliac Perforation Using a Viabahn Endoprosthesis during Transfemoral Aortic Valve Implantation
}

Noritoshi Ito, Tetsuya Tobaru, Kei Hatori, Yu Asukai, Shinya Wada, Hojo Sasaki, Hideo Takahashi, Naoki Saito, Toru Kawakami, Hiroshi Fukunaga, Hiroki Ogura, Kazuya Kumagai, Kenji Wada, Takahide Yoshio, Tomoya Uchimuro, and Shuichiro Takanashi

Kawasaki Saiwai Hospital, Cardiovascular Center, Kawasaki, Kanagawa, Japan

Received: September 2, 2020; Accepted: March 5, 2021

Objective: Here, we report the strategy of bailout in case of transfemoral-transcatheter aortic valve implantation (TFTAVI)-induced iliac arterial injury during TAVI.

Case Presentation: We show a case of a 79-year-old male who presented with severe symptomatic aortic stenosis. We performed TAVI from the left common femoral artery. We inserted a 14-Fr e-sheath without difficulty, and we delivered and deployed successfully a 26-mm SAPIEN3-valve (Edwards Lifesciences, Irvine, CA, USA). When the 14-Fr e-sheath was removed, the aortography showed massive extravasation from the left iliac artery. As a result, we were able to rapidly treat the iliac perforation using Viabahn endoprostheses and less invasively than a conventional stent-graft or surgical repair while obtaining good patency with the Viabahn endoprostheses.

Conclusion: We had an experience that the use of Viabahn endoprostheses is safe and effective in case of TF-TAVI-induced iliac arterial injury during TAVI, with good short- and mid-term outcomes.

Keywords: transcatheter aortic valve implantation (TAVI), aortic stenosis, transfemoral (TF) approach, vascular access complications (VACs), Viabahn endoprostheses

\section{Introduction}

Transcatheter aortic valve implantation (TAVI) was incubated to treat elderly patients with severe symptomatic aortic stenosis at high risk for surgery, and it is most commonly performed with a retrograde transfemoral (TF) approach. The TF approach is the first choice in most centers performing TAVI. A percutaneous TAVI procedure has the potential to shorten procedure duration, reduce the risk of wound infection, and keep patient immobilization and discomfort and length of hospitalization to a

Corresponding author: Tetsuya Tobaru. Kawasaki Saiwai Hospital, Cardiovascular Center, 31-27, Omiya-cho, Saiwai-ku, Kawasaki, Kanagawa 212-0014, Japan

Email: ttobaru9@gmail.com

This work is licensed under a Creative Commons Attribution-NonCommercialNoDerivatives International License.

C2021 Japan Transcatheter Valve Therapies minimum, compared with a surgical cutdown to the femoral artery. ${ }^{1-3)}$ However, in up to $30 \%$ of cases, it is either not recommended to choose TF approach or the patient is considered to be at high risk of vascular access complications (VACs), which is a major cause of morbidity and mortality. ${ }^{4,5}$ The use of self-expanding covered stents as a treatment option for vascular injuries during TAVI has been described previously. ${ }^{2-6)}$

\section{Case Report}

This is a case of a 79-year-old male (body mass index of $23.6 \mathrm{~kg} / \mathrm{m}^{2}$ ) who presented with severe symptomatic aortic stenosis and an aortic valve area of $0.60 \mathrm{~mm}^{2}$ and mean gradient of $40 \mathrm{mmHg}$ on echocardiography. He was admitted to our hospital with acute heart failure due to severe aortic stenosis. His past medical history includes hypertension, dyslipidemia, and chronic renal failure (estimated glomerular filtration rate: $34.8 \mathrm{~mL} / \mathrm{min} / 1.73 \mathrm{~mm}^{2}$ ). 
He was deemed moderate risk with an the Society of Thoracic Surgeons (STS) score of 5.14\% and a clinical frailty score of 3 . He refused surgical aortic valve replacement and was expected to have TAVI. On the pre-procedural contrast CT, his left iliac artery was $5.8 \mathrm{~mm}$ and right iliac artery was $4.3 \mathrm{~mm}$ at a minimum, and there was little atherosclerotic change (Fig. 1). We performed TF-TAVI from the left side. We inserted a 14-Fr e-sheath without difficulty. A 26-mm SAPIEN3-valve (Edwards Lifesciences, Irvine, CA, USA) was delivered and deployed successfully (Fig. 2). When the 14-Fr e-sheath was removed, the aortography showed massive extravasation from the left iliac artery (Fig. 3A). We inserted the 14-Fr sheath and dilated an 8-mm-balloon immediately for hemostasis, and a VIABAHN Endoprosthesis (Gore, Flagstaff, AZ, USA) $(10-\mathrm{mm} \times 10-\mathrm{cm})$ was retrogradely inserted covering the injured left iliac artery using a 0.018-inch stiff-guidewire. After dilating the VIABAHN Endoprosthesis with an 8-mm balloon, complete hemostasis was obtained for the iliac extravasation (Fig. 3B). He was discharged on postoperative day 11 without sequelae. His general condition has been good without circulatory complications to his lower limb for 6 months after discharge.

\section{Discussion}

According to De Backer et al, ${ }^{7)}$ when 354 patients received percutaneous TF-TAVI, 72 patients $(20.3 \%)$ had VACs that

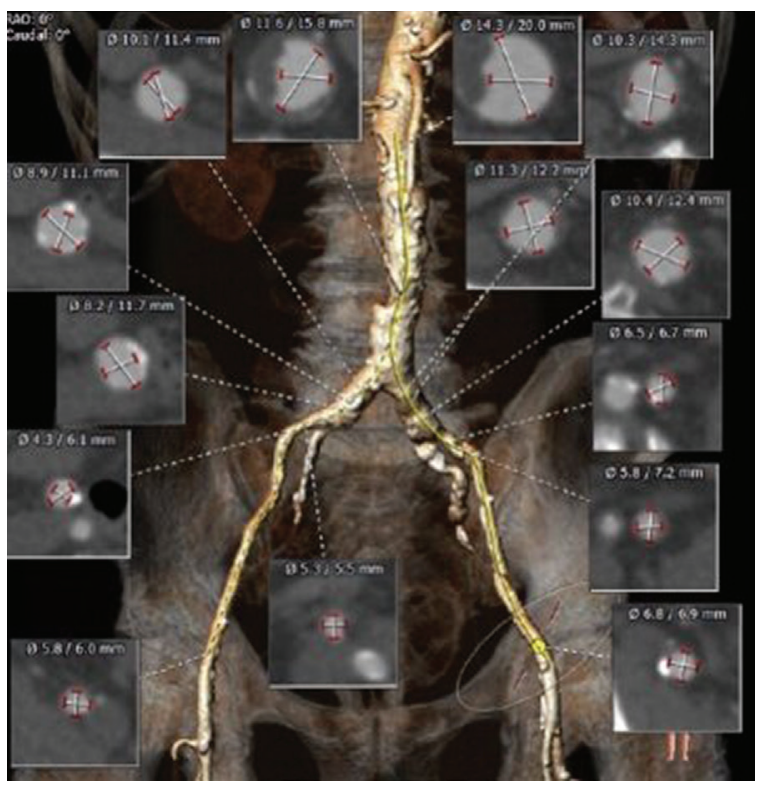

Fig. 1 Measurement of the iliac artery on pre-procedural computed tomography required acute intervention. Of these, 18 patients received balloon angioplasty, 48 patients received a Viabahn endoprosthesis, and 6 patients required surgical vessel repair. Interesting to note, follow-up with color duplex US showed $100 \%$ patency of the Viabahn endoprostheses with no cases of stent fracture or in-stent stenosis or occlusion.

It would be fatal without the appropriate treatment for massive extravasation from the left iliac artery. The e-sheath for SAPIEN3-valve temporarily enlarges by unfolding to allow entry of the device (expanded outer diameter $8 \mathrm{~mm}$ for the 26-mm SAPIEN3 valve). ${ }^{8}{ }^{8}$ It might cause massive extravasation from the iliac artery. In this case, although we could not expect this complication based on the pre-procedural contrast CT, we were able to rapidly treat the iliac perforation using Viabahn endoprostheses and less invasively than a conventional stentgraft or surgical repair while obtaining good patency with the Viabahn endoprostheses. The important point is that when the 14-Fr e-sheath was removed, we should leave a 0.035 inch stiff-guidewire to make it easy to deliver Viabahn endoprostheses, not a 0.035 inch soft-guidewire.

\section{Conclusion}

We had an experience that the use of Viabahn endoprostheses is safe and effective in case of TF-TAVI-induced iliac arterial injury during TAVI, with good short- and mid-term outcomes.

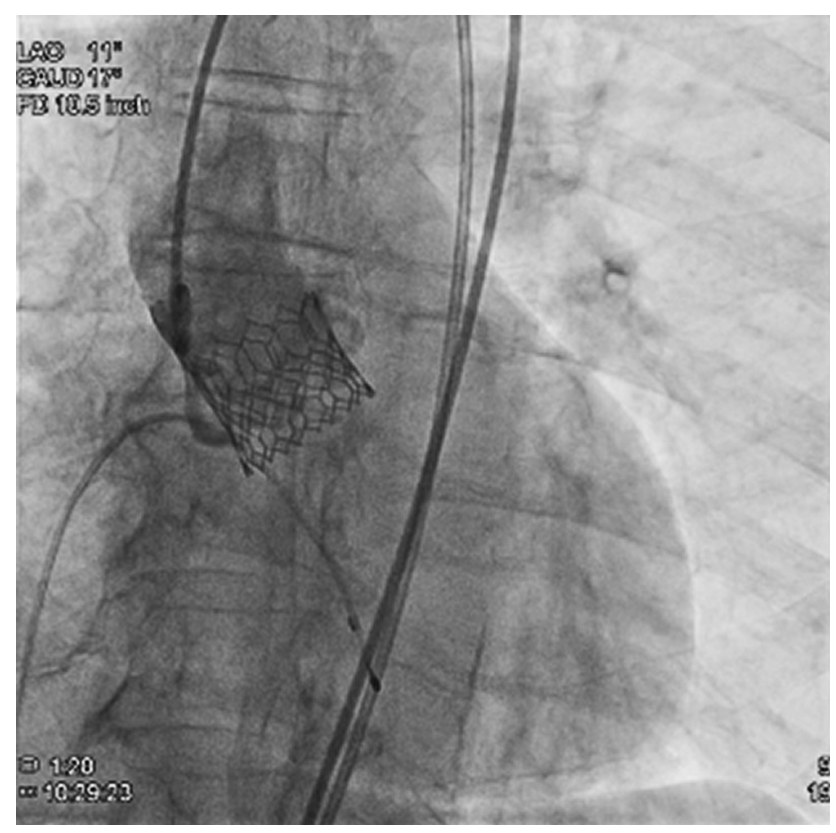

Fig. 2 Successful deployment of a 26-mm SAPIEN3-valve 

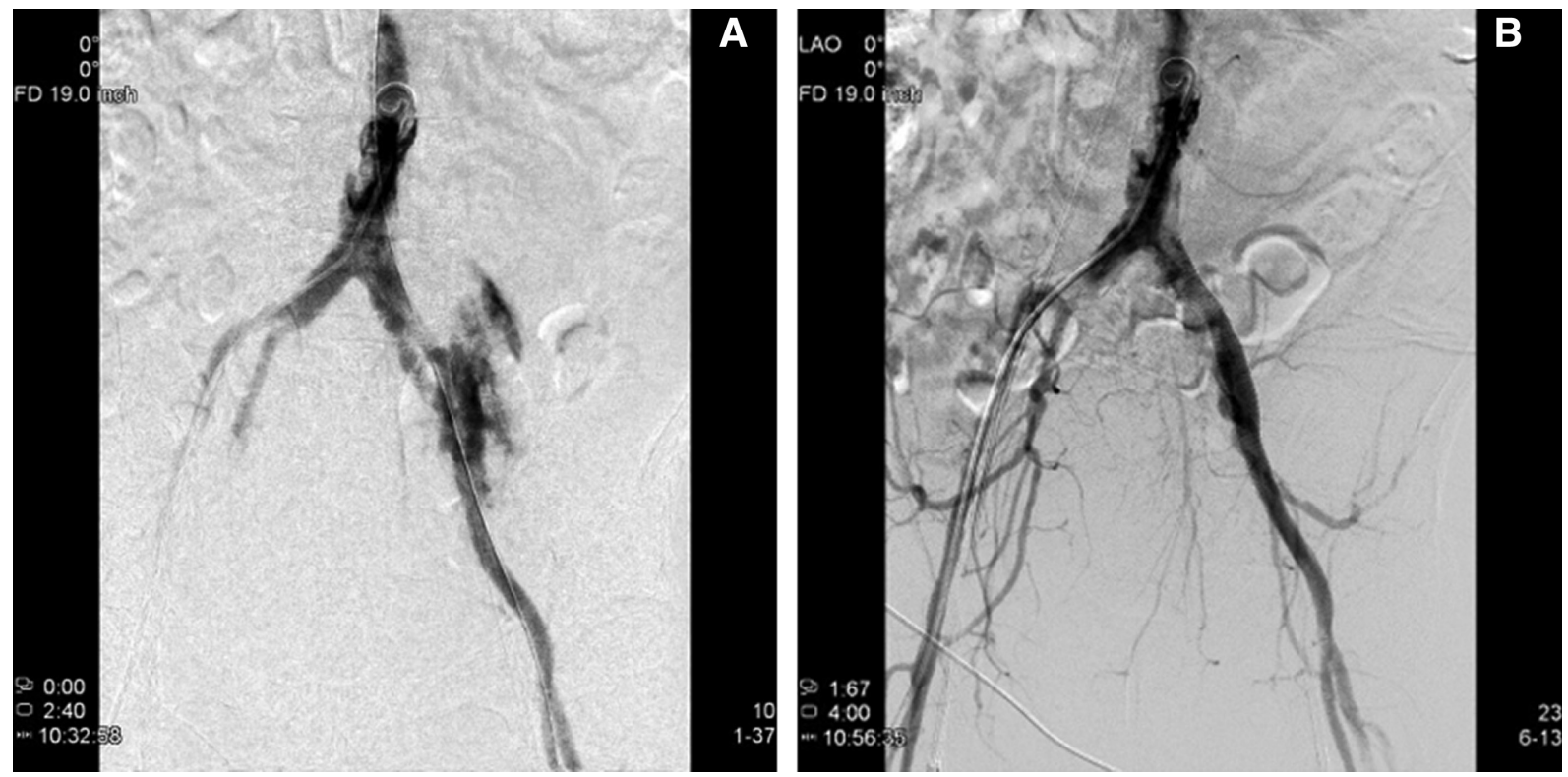

Fig. 3 (A) Massive extravasation from the left iliac artery perforation and (B) after stabilization of the iliac artery perforation

\section{Author Contribution}

The conception and design of the work: N. Ito and T. Tobaru

The acquisition and analysis of data for the work: N. Ito and K. Hatori

Drafting the work: N. Ito

The interpretation of data for the work, revising the work critically for important intellectual content: T. Tobaru and K. Hatori

Final approval of the version to be published: all authors

Accountability for all aspects of the work: all authors

\section{Disclosure Statement}

Dr. Tobaru is the proctor of TAVI for Edwards Lifesciences and Medtronic. The other authors have no conflicts of interest directly relevant to the content of this article.

\section{References}

1) Schäfer U, Ho Y, Frerker C, et al: Direct percutaneous access technique for transaxillary transcatheter aortic valve implantation: "the Hamburg Sankt Georg approach". JACC Cardiovasc Interv 2012; 5: 477-486.
2) Nakamura M, Chakravarty T, Jilaihawi H, et al.: Complete percutaneous approach for arterial access in transfemoral transcatheter aortic valve replacement: a comparison with surgical cut-down and closure. Catheter Cardiovasc Interv 2014; 84: 293-300.

3) Hayashida K, Lefèvre T, Chevalier B, et al: True percutaneous approach for transfemoral aortic valve implantation using the Prostar XL device: impact of learning curve on vascular complications. JACC Cardiovasc Interv 2012; 5: 207-214.

4) Toggweiler S, Leipsic J, Binder RK, et al: Management of vascular access in transcatheter aortic valve replacement: part 2: vascular complications. JACC Cardiovasc Interv 2013; 6: 767-776.

5) Toggweiler S, Webb JG: Challenges in transcatheter aortic valve implantation. Swiss Med Wkly 2012; 142: w13735.

6) Kojima Y, Higuchi R, Saji M, et al: Complete endovascular repair of iliac artery perforation during transcatheter aortic valve implantation: a bailout with Viabahn endoprosthesis. Cardiovasc Interv Ther 2020; 35: 205-206.

7) De Backer O, Arnous S, Sandholt B, et al: Safety and efficacy of using the Viabahn endoprosthesis for percutaneous treatment of vascular access complications after transfemoral aortic valve implantation. Am J Cardiol 2015; 115: 1123-1129.

8) Binder RK, Rodés-Cabau J, Wood DA, et al: Transcatheter aortic valve replacement with the SAPIEN 3: a new balloon-expandable transcatheter heart valve. JACC Cardiovasc Interv 2013; 6: 293-300. 